\title{
Prevalence and molecular basis of glucose-6- phosphate dehydrogenase deficiency in Afghan populations: implications for treatment policy in the region
}

Toby Leslie ${ }^{1,2^{*}}$, Bushra Moiz ${ }^{3}$, Nader Mohammad ${ }^{1}$, Omar Amanzai ${ }^{1}$, Haroon ur Rasheed ${ }^{1}$, Sakhi Jan ${ }^{1}$, Abdul M Siddiqii, Amna Nasir ${ }^{3}$, Mohammad A Beg $^{3}$ and Martijn Vink ${ }^{4}$

\begin{abstract}
Background: Glucose-6-phosphate dehydrogenase deficiency (G6PD), an x-linked inherited enzymopathy, is a barrier to malaria control because primaquine cannot be readily applied for radical cure in individuals with the condition. In endemic areas, including in Afghanistan, the G6PD status of vivax patients is not routinely determined so the drug is rarely, if ever, prescribed even though it is included as a recommended treatment in local, regional and global guidelines. This study assessed the prevalence and genotype of G6PD deficiency in Afghan populations and examined the need for routine G6PD testing as a malaria treatment and control tool.

Methods: A cross-sectional household survey was conducted using random sampling in five Afghan cities to determine the prevalence of G6PD deficiency in Afghan ethnic groups. Filter-paper blood spots were analysed for phenotypic G6PD deficiency using a fluorescent spot test. Molecular analysis was conducted to identify the genetic basis of the disorder.

Results: Overall, 45/1,436 (3.1\%) people were G6PD deficient, 36/728 (5.0\%) amongst males and 9/708 (1.3\%) amongst females. Amongst males the prevalence was highest in the Pashtun ethnic group $(10 \%, 26 / 260)$ while in Tajik males it was 8/250 (3.2\%); in Hazara males it was 1/77 (1.3\%) and in Uzbek males is was 0/125. Genetic testing in those with deficiency showed that all were of the Mediterranean type (Med-) characterized by a C-T change at codon 563 of the G6PD gene.

Conclusion: Prevalence of G6PD deficiency in Afghanistan varies considerably by ethnic group and is predominantly of the Mediterranean type. G6PD deficient individuals are susceptible to potentially severe and lifethreatening haemolysis after standard primaquine treatment. If the aim of increasing access to radical treatment of vivax is to be successful reliable G6PD testing needs to be made routinely available within the health system.
\end{abstract}

Keywords: Malaria, G6PD, Glucose-6-phosphate dehydrogenase deficiency, Afghanistan, Primaquine, Vivax, Relapse

\footnotetext{
* Correspondence: toby.leslie@lshtm.ac.uk

${ }^{1}$ HealthNet TPO, Kabul, Afghanistan

${ }^{2}$ London School of Hygiene and Tropical Medicine, London, UK

Full list of author information is available at the end of the article
} 


\section{Background}

Glucose-6-phosphate dehydrogenase (G6PD) deficiency is the most common genetic enzyme disorder in humans [1]. Worldwide, approximately 400 million people carry the deficiency gene and over 140 G6PD variants have been genetically characterized. The worldwide distribution closely matches that of malaria and provides some protection, suggesting that the disease has exerted evolutionary pressure for retention of the trait in humans [2]. Because the G6PD gene is located on the Xchromosome, the prevalence of G6PD in males is higher than that of females [1]. The deficiency can cause haemolytic episodes ranging from subclinical and mild to severe and fatal in response to stressors which include commonly used drugs. The G6PD deficiency occurring in $\sim 10 \%$ of men of African descent (African (A-) variant) is regarded as milder than other forms although fatal reactions to primaquine have been recorded in a limited number of patients [3]. The Mediterranean variant (Med-) is regarded as more severe.

Although commonly asymptomatic, G6PD-deficiency becomes clinically relevant in response to oxidative stress [4] including from the anti-malarial drug, primaquine [5]. Red cells of those with severe deficiency are susceptible to haemolysis when exposed to therapeutic doses of the drug.

Primaquine is the only licensed medicine for treatment of the dormant liver stage of Plasmodium vivax. The parasite's ability to form latent hynozoites in infected individuals causes relapse episodes for months or years following the initial infection without the bite of an infective mosquito. The hynozoite is therefore responsible for a large proportion of vivax malaria cases and is the principle disease reservoir.

In Afghanistan, as in most of Asia, malaria is caused predominantly by $P$. vivax but the unknown G6PD status in most patients guarantees that almost none receive adequate drug therapy for radical cure of confirmed vivax malaria. This situation is similar in most areas of south Asia, where G6PD testing is not routinely applied, representing a major impediment to control of the disease and a failure of policy over practice.

No systematic estimates of G6PD deficiency prevalence exists for Afghanistan. In order to inform decision making on the need for G6PD testing in the health system, a cross-sectional study was undertaken to estimate the prevalence of G6PD deficiency in the population and to characterize the G6PD deficiency variants.

\section{Methods}

The primary objective of the study was to estimate the prevalence of G6PD deficiency in the population of Afghanistan. The secondary objectives were to estimate the prevalence of G6PD deficiency among different ethnics groups in Afghanistan and to identify the G6PD deficiency variants in the population.

\section{Sample selection}

A cross-sectional study design was used encompassing five major Afghan cities. Afghanistan is ethnographically diverse and the study settings were applied to reflect this diversity. The most populous ethnic groups are Pashtun and Tajik with fewer from Hazara, Uzbek and Turkmen groups and small minorities of Pashaei, Noristani, Arab, and mixed ethnic groups

The study was conducted in major urban centres of five Afghan provinces: Nangarhar (Jalalabad), Takhar (Taloquan), Faryab (Maimana), Bamyan (Bamyan) and Kabul (Kabul City).

Nangarhar is located in the east of the country and has the highest intensity malaria transmission of the study sites (ten to 100 cases per 1,000 persons per year). The province is dominated by Pashtun ethnicity, but two minority ethnic groups, Arabs and Pashaees, are also settled in Nangarhar. The north-eastern province of Takhar is endemic for malaria, but at lower levels than in the east and has predominantly Tajik and Uzbek ethnicity. The north-western province of Faryab, endemic for malaria with very low transmission levels $(<1 / 1,000$ per year) and contains Turkmen, Uzbek and Tajik ethnic groups. Bamyan's population in the Central Highlands of the country is predominantly of Hazara ethnic group, and Kabul is the multi-ethnic capital city of the country with all groups represented. Neither Bamyan nor Kabul is endemic for malaria.

The sample sites, therefore, reflect the majority of ethnic groups in Afghanistan and are readily accessible in terms of security, geography and resource availability. The sample was not intended to (and does not) estimate the relative proportion of each ethnic group in the population as a whole.

To detect a prevalence of G6PD deficiency $>3 \%$ in the population [6], with $90 \%$ power at the $95 \%$ confidence level, 942 persons were required. To establish reasonably precise estimates within individual ethnic groups and to account for differences by gender required a larger sample size of 1,500 . The sample collected from each city was proportionate to relative populations (Table 1).

Households were selected using random transect lines drawn within the municipal limits on maps of each city [7]. Each transect was used to identify households, selecting every third house and then every eighth, $13^{\text {th }}$, etc. house (using a sampling interval of five), until the required number of households were recruited. Two persons per household (one male and one female) were selected. Additional transects were used in the same way if too few houses were available on the initial transect. 
Table 1 Population and proportion in the sample

\begin{tabular}{llll}
\hline & Total $^{*}$ & \% of total & Number in study \\
\hline Jalalabad & $1,289,000$ & 20 & 297 \\
Kabul & $3,138,000$ & 48 & 722 \\
Maimana & 858,000 & 13 & 197 \\
Taloquan & 845,000 & 13 & 194 \\
Bamyan & 387,000 & 6 & 89 \\
TOTAL & $6,517,000$ & 100 & 1,500 \\
\hline
\end{tabular}

* Data from Central Statistics Office 1385 (2007).

One male and one female volunteer who matched the inclusion and none of the exclusion criteria were selected. The sample included any ethnic group living in the study household; males and females; and those aged over three years. Participants were excluded if they refused to give consent; were under three years of age; were incompetent to give consent; or were infirm. A simple pretested questionnaire was used for data collection. Informed consent was taken at enrolment into the study. Ethical approval was given by the Institutional Review Board of the Ministry of Public Health of Afghanistan.

\section{Detection of G6PD deficiency}

Trained surveyors collected filter paper blood spots (Whatman 3M filter papers) marked with the patient number and stored in sealed bags with silica beads. Samples were processed on the following day.

G6PD deficiency status was based on a fluorescent spot test (FST) (PishtazTeb Diagnostics, Tehran, Islamic Republic of Iran) to identify the production of nicotinamide adenine dinucleotide phosphate-oxidase (NADPH) from nicotinamide adenine dinucleotide phosphate (NADP) [8]. The FST is a standard qualitative test for the detection of G6PD deficiency and can be performed in about $15 \mathrm{~min}$. The test is considered positive (i.e. a patient is deficient) when the blood spot fails to fluoresce under ultraviolet light (Figure 1).

Genetic analysis of the samples was undertaken at The Aga Khan University Hospital, Karachi, Pakistan using the filter paper blood-spots collected in the field. Due to resource constraints, a subsample was selected to include all samples with detected deficiency and, by gender, $10 \%$ of negative samples from male participants and $50 \%$ of all female samples. G6PD non-deficient subjects were included to confirm the G6PD screening test and to identify the proportion of females carrying heterozygous copies of the gene, which are not detected by the fluorescent spot test.

Genomic DNA was extracted from dried blood spots using mini Qiagen ${ }^{\circ}$ DNA Isolation kit (Qiagen Inc, Chatsworth, CA, USA) according to the manufacturer's

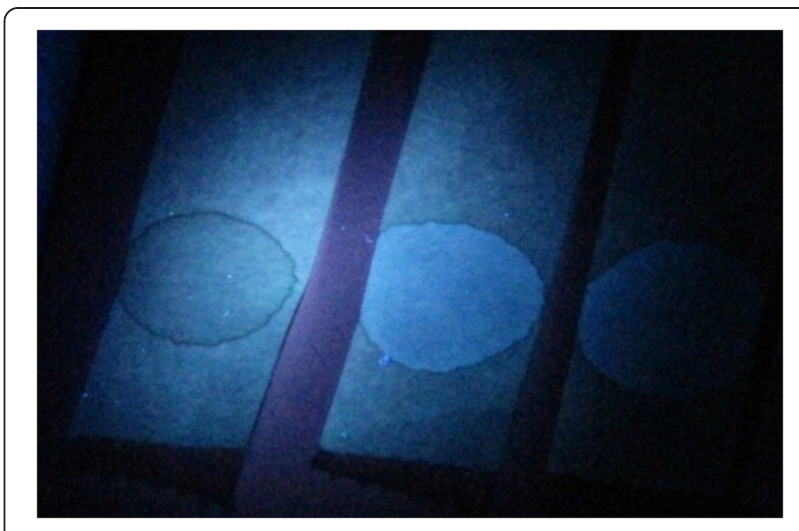

Figure $1 \mathrm{G} 6 \mathrm{PD}$ fluorescent spot test under ultraviolet light (365nm) (Beutler test) [8]. The left filter paper shows severe deficiency, and the two on the right show a normal reaction.

instructions. DNA was eluted in $50 \mathrm{ul}$ of elution buffer and stored at $-20^{\circ} \mathrm{C}$ until use.

Subsequently the DNA was employed as a template in a PCR reaction which used two gene specific primers and the reagents required for DNA amplification. The obtained amplifiers were subjected to overnight restriction enzyme digestion followed by separation of the digested products on an agarose gel electrophoresis for detecting the presence or absence of specific point mutations. Initially all samples were examined for the 563C-T mutation of the Med- allele. Those remaining uncharacterized were analysed for 1003 A-G (Chatham) and 131 C-G (Orissa) [9]. The primers and restriction enzymes were utilized according to previously established protocols.

\section{Data analysis}

After correction and coding, the complete dataset was double entered by two data entry operators using Epiinfo, version 6.04. The two datasets were compared for data consistency and for missing values. Analysis was done by using STATA v8. The primary outcome is the proportion of people in the sample with phenotypic G6PD deficiency, as measured by the fluorescent spot test. The results were also stratified by the explanatory variables of ethnic group, gender and province (study site). Additional analysis was conducted on the genetic data to identify the proportion of males who were hemizygous deficient and the proportion of females who were homozygous deficient, non-deficient (wild-type) and heterozygous deficient, cross-tabulated against the FST result.

\section{Results}

\section{Sample characteristics}

In total, 1,500 individuals were enrolled in the study during July and August 2010. Data from 64 (4.3\%) were 
excluded from the analysis for missing data (one missed the G6PD test result, 45 missed gender, ten missed ethnicity data, eight had age inaccurately recorded). Data from 1,436 participants were evaluated.

The sample characteristics are shown in Table 2. Mean age was 23 years, and $45 \%$ of the population was under 15 years, matching the age demographic for Afghanistan. Half of the sample was male.

All of the majority ethnic groups are included in the sample and most of the minority ethnic groups were also identified. For the main analysis the minority ethnic groups of Turkmen $(n=2)$, Arab $(n=10)$, Pashaee $(n=22)$ and Noristani $(n=4)$ have been grouped together because too few were in the sample for adequate analysis. The proportion of participants in each ethnic group does not appear to differ from widely available estimates of the ethno-linguistic composition of the country (e.g. [10]). The proportion of participants from each of the five provinces matches the relative size of the total provincial population.

\section{G6PD deficiency prevalence}

Amongst the sample, 45 (3.1\%) participants were phenotypically deficient for G6PD. Because the deficiency is Xlinked, males and females were analysed separately. G6PD deficiency was found in 36/728 (5.0\%) of males and 9/708 (1.3\%) of females $\left(\mathrm{chi}^{2}=16.0, \mathrm{p}<0.001\right)$.

Prevalence of deficiency differed by ethnic group. Amongst both males and females G6PD deficiency was more frequent in Pashtuns than in other ethnic groups,

Table 2 Characteristics of the sample

\begin{tabular}{ll}
\hline Total evaluable participants & 1,436 \\
Mean age & 23.0 years \\
$\%$ age $<15$ years & 45.7 \\
$\%$ male & 50.7 \\
Ethnic group, n (\%): & \\
Pashtun & $519(36.1)$ \\
Tajik & $490(34.1)$ \\
Uzbek & $238(16.6)$ \\
Hazara & $152(10.6)$ \\
Turkmen & $2(0.1)$ \\
Arab & $10(0.7)$ \\
Pasheaa & $21(1.5)$ \\
Noristani & $4(0.3)$ \\
Province (city name), n (\%): & \\
Bamyan & $89(6.2)$ \\
Faryab (Maimana) & $180(12.5)$ \\
Kabul & $688(47.9)$ \\
Nangahar (Jalalabad) & $295(20.5)$ \\
Takhar (Taloquan) & $184(12.8)$ \\
\hline &
\end{tabular}

and in Pashtun males deficiency was 10\% (26/260) (Table 3).

The frequency of G6PD deficiency did not differ by age group, as expected from a genetically inherited trait, but it did differ by province due to the differing ethnic make-up of each province.

The proportion of respondents who had a selfreported history of jaundice was higher amongst G6PDdeficient individuals than non-deficient individuals $(7 / 45$ [15.6\%] vs 88/1,391 [6.3\%]) (Fisher's exact test, $\mathrm{p}=0.038$ ). There was no difference in the proportion of deficient or non-deficient participants who had had an anti-malarial, had self-reported malaria or who had ever been admitted to hospital.

Mutational analysis was successfully conducted on 480/500 subjects as follows: $44 / 45$ samples identified as G6PD deficient by the fluorescent spot test [34M/10F], a random selection of 378 G6PD normal females and 67 G6PD normal males.

Table 4 shows the number and proportion of participants identified by genetic analysis and the fluorescent spot test. Sensitivity and specificity of the fluorescent spot-test among specimens from males was 89.2 and $98.4 \%$, respectively. For females, in differentiation of homozygous from wild type, the sensitivity and specificity of the fluorescent spot test was 72.7 and $99.7 \%$. Only one of the 34 heterozygous females was detected by the fluorescent spot test.

\section{Conclusions}

G6PD deficiency is prevalent in the Afghan population and differs between ethnic groups. In male Pashtuns the prevalence of the deficiency is $10 \%$ and amongst female Pashtuns, 2\%. Other ethnic groups also showed an appreciable prevalence.

The findings in this study are consistent with other studies in the region. In one such study [6] in Pashtun Afghan refugees in Pakistan, the prevalence of G6PD deficiency was about $10 \%$ amongst males. Bouma et al. [11] found that the prevalence of G6PD deficiency varied within the Pashtun population; amongst male Afghan

Table 3 Prevalence of G6PD deficiency amongst males and females in different ethnic groups of Afghanistan [n/N (\%)]

\begin{tabular}{lll}
\hline Ethnicity & Males* $^{*}$ & Females \\
\hline Pashtun & $26 / 260(10.0 \%)$ & $6 / 257(2.3 \%)$ \\
Tajik & $8 / 244(3.2 \%)$ & $2 / 244(0.8 \%)$ \\
Uzbek & $0 / 125$ & $0 / 113$ \\
Hazara & $1 / 79(1.3 \%)$ & $1 / 73(1.4 \%)$ \\
Other & & $1 / 20(5.0 \%)$ \\
Total & $2 / 37(5.4 \%)$ & $10 / 712(1.3 \%)$ \\
\hline
\end{tabular}

${ }^{*} \mathrm{Chi}^{2}=24.5, \mathrm{p}<0.001$ (4d.f.); **Turkmen, Arab, Pashaee, Nooristani. 
Table 4 G6PD c.563 C>T variants in 44 G6PD-deficient and 436 non-deficient subjects

\begin{tabular}{|c|c|c|c|c|c|}
\hline \multirow[b]{3}{*}{ FST result } & \multicolumn{5}{|c|}{ Genetic testing results (PCR) } \\
\hline & \multicolumn{2}{|l|}{ Male } & \multicolumn{3}{|l|}{ Female } \\
\hline & haemizygous deficient & Wild type & Homozygous & Heterozygous & Wild type \\
\hline Non-deficient & $4(10.8)$ & 60 & $3(27.2)$ & 33 & 336 \\
\hline Deficient & 33 & $1(1.7)$ & 8 & 1 & $1(0.3)$ \\
\hline
\end{tabular}

pashtuns, prevalence was $15.8 \%$ while amongst male Pakistani pashtuns it was less (7\%), although there was no statistical difference between the two groups. Any differences could be explained by the existence of many subgroups of Pashtun tribes [12] with intra-ethnic variance amongst these subgroups [13]. This may explain why Ali et al. [13] found a lower G6PD prevalence of $3.2 \%$ in ethnic Pashtun males in Pakistan.

Rehbolz et al. [14] found a prevalence of $2.1 \%$ in Tajikistan, and $1.4 \%$ amongst males of the Tajik ethnic group, consistent with the prevalence found in this study. The findings greatly differ for the Uzbek population, however. This study found zero amongst 238 tested ethnic Uzbek participants, where the study in Tajikistan [14] identified a prevalence of $4.2 \%$ and Bouma et al. found a prevalence of $9.1 \%$ among male Afghan refugees of Uzbek ethnicity in Pakistan [11]. This may be due to differences in reporting of ethnicity by the population under study as the parental lineage may be mixed.

The genetic analysis confirmed that almost all deficiency in this region is caused by the Med- allele. This variant has been associated with moderate to severe haemolytic reactions to primaquine. Testing for G6PD deficiency that is cheap and reliable is therefore required. Although one study indicates that the risk of malaria infection is lower in G6PDdeficient individuals [6] with an $85 \%$ protective effect, it is likely that around 1 or $2 \%$ patients will be deficient. The risk of exposing G6PD-deficient patients to severe complications may be lower than assumed but, crucially, is not zero.

The choice of test is an important factor and far from straightforward. The inaccuracy of the FST in this study in male patients could lead to false "normal" results. Detection of deficiency in females is difficult because female heterozygotes exhibit mozaicism and varying proportions of G6PD-deficient and non-deficient red cells circulate concurrently. Detecting heterozygous females is not possible with the phenotypic FST unless the patient is significantly deficient. The clinical implications of this are not clear [15]. A different cytochemical test may be employed for female patients, but is more expensive that the simple FST and requires more sophisticated laboratories. Prototype rapid tests show promise but provided few, though significant, false "normal" results [16].

The implications of an accurate and reliable test being widely used to improve radical cure are clear. The hypnozoite reservoir provides the means for the disease to transmit across the seasons - a peak of relapse episodes in the early season coincides with the bloom of mosquito vectors which is a characteristic of long latency subtropical strains of vivax. Relapses also render control interventions aimed at reducing vector-human contact less effective because episodes occur without the bite of a vector [17]. If the majority of patients could be treated with primaquine then the effect on transmission in areas of seasonal malaria could be dramatic. In G6PD-normal Afghan Pashtuns with vivax malaria in Pakistan who were treated with 14-day primaquine in a randomized trial, $2 \%$ of patients had one subsequent episode of vivax over 11 months of follow-up, while in the untreated group it was 30\% $[18,19]$

Routine use of primaquine, as recommended in WHO and local guidelines, in all vivax patients would be a valuable control and elimination tool if applied to a high proportion of the population but this strategy requires accurate and reliable G6PD testing to be routinely available. If successfully employed, routine G6PD testing could result in significant reductions in transmission of vivax malaria.

\section{Competing interests}

The authors declare that they have no competing interests.

\section{Authors' contributions}

TL, NM, HR, MS, OA and SJ designed the study and conducted the field work; TL conceived the study; TL and NM analysed the data; TL, NM and MV wrote the paper; $B M, A M$ and $A B$ conducted the genetic analysis and contributed to the paper. All authors provided intellectual input into this paper and read and approved the final version.

\section{Acknowledgements}

This study was sponsored by HealthNet TPO with funding from the Global Fund for AIDS TB and Malaria, Round 8. The authors are grateful to the participants, the Ministry of Public Health (National Malaria and Leishmaniasis Control Programme) and to Amy Mikhail (for advice on field laboratory testing).

\section{Author details}

${ }^{1}$ HealthNet TPO, Kabul, Afghanistan. ${ }^{2}$ London School of Hygiene and Tropical Medicine, London, UK. ${ }^{3}$ Aga Khan University, Karachi, Pakistan. ${ }^{4}$ HealthNet TPO, Amsterdam, The Netherlands.

Received: 25 April 2013 Accepted: 1 July 2013

Published: 8 July 2013

\section{References}

1. Beutler E, Duparc S: Glucose-6-phosphate dehydrogenase deficiency and antimalarial drug development. AmJTrop Med Hyg 2007, 77:779-789. 
2. Howes RE, Piel FB, Patil AP, Nyangiri OA, Gething PW, Dewi M, Hogg MM, Battle KE, Padilla CD, Baird JK, Hay SI: G6PD Deficiency prevalence and estimates of affected populations in malaria endemic countries: a geostatistical model-based map. PLoS Med 2012, 9:e1001339.

3. Lacerda MV, Fragoso SC, Alecrim MG, Alexandre MA, Magalhaes BM, Siqueira AM, Ferreira LC, Araujo JR, Mourao MP, Ferrer M, Castillo P, MartinJaular L, Fernandez-Becerra C, del Portillo H, Ordi J, Alonso PL, Bassat Q: Postmortem characterization of patients with clinical diagnosis of Plasmodium vivax malaria: to what extent does this parasite kill? Clin Infect Dis 2012, 55:e67-74.

4. Cappellini MD, Fiorelli G: Glucose-6-phosphate dehydrogenase deficiency. Lancet 2008, 371:64-74.

5. Alving AS, Carson PE, Flanagan CL, Ickes CE: Enzymatic deficiency in primaquine-sensitive erythrocytes. Science 1956, 124:484-485.

6. Leslie T, Briceno M, Mayan I, Mohammed N, Klinkenberg E, Sibley CH, Whitty $\mathrm{CJ}$, Rowland M: The impact of phenotypic and genotypic G6PD deficiency on risk of plasmodium vivax infection: a case-control study amongst Afghan refugees in Pakistan. PLoS Med 2010, 7:e1000283.

7. Afghanistan Information and Management Systems. www.aims.org.af.

8. Beutler $\mathrm{E}$, Mitchell M: Special modifications of the fluorescent screening method for glucose-6-phosphate dehydrogenase deficiency. Blood 1968, 32:816-818.

9. Minucci A, Moradkhani K, Hwang MJ, Zuppi C, Giardina B, Capoluongo E: Glucose-6-phosphate dehydrogenase (G6PD) mutations database: review of the "old" and update of the new mutations. Blood Cells Mol Dis 2012, 48:154-165.

10. Library of Congress: Country Series - Afghanistan. http://lcweb2.loc.gov/ $\mathrm{frd} / \mathrm{cs} / \mathrm{aftoc} . \mathrm{htm}$

11. Bouma MJ, Goris M, Akhtar T, Khan N, Kita E: Prevalence and clinical presentation of glucose-6-phosphate dehydrogenase deficiency in Pakistani Pathan and Afghan refugee communities in Pakistan; implications for the use of primaquine in regional malaria control programmes. Trans R Soc Trop Med Hyg 1995, 89:62-64.

12. Caroe O: The Pathans: 500BC-AD1957. Oxford: Oxford University Press; 1984

13. Ali N, Anwar M, Ayyub M, Bhatti FA, Nadeem M, Nadeem A: Frequency of glucose-6-phosphate dehydrogenase deficiency in some ethnic groups of Pakistan. J Coll Physicians Surg Pak 2005, 15:137-141.

14. Rebholz CE, Michel AJ, Maselli DA, Saipphudin K, Wyss K: Frequency of malaria and glucose-6-phosphate dehydrogenase deficiency in Tajikistan. Malar J 2006, 5:51.

15. Peters AL, Van Noorden CJ: Glucose-6-phosphate dehydrogenase deficiency and malaria: cytochemical detection of heterozygous G6PD deficiency in women. J Histochem Cytochem 2009, 57:1003-1011.

16. Kim S, Nguon C, Guillard B, Duong S, Chy S, Sum S, Nhem S, Bouchier C, Tichit M, Christophel E, Taylor WR, Baird JK, Menard D: Performance of the CareStart G6PD deficiency screening test, a point-of-care diagnostic for primaquine therapy screening. PLoS One 2011, 6:e28357.

17. Rowland M, Bouma M, Ducornez D, Durrani N, Rozendaal J, Schapira A, Sondorp E: Pyrethroid-impregnated bed nets for personal protection against malaria for Afghan refugees. Trans R Soc Trop Med Hyg 1996, 90:357-361.

18. Leslie T, Mayan I, Mohammed N, Erasmus P, Kolaczinski J, Whitty CJ, Rowland M: A randomised trial of an eight-week, once weekly primaquine regimen to prevent relapse of Plasmodium vivax in Northwest Frontier Province, Pakistan. PLoS One 2008, 3:e2861.

19. Leslie T, Rab MA, Ahmadzai H, Durrani N, Fayaz M, Kolaczinski J, Rowland M: Compliance with 14-day primaquine therapy for radical cure of vivax malaria-a randomized placebo-controlled trial comparing unsupervised with supervised treatment. Trans R Soc Trop Med Hyg 2004, 98:168-173.

doi:10.1186/1475-2875-12-230

Cite this article as: Leslie et al.: Prevalence and molecular basis of glucose-6-phosphate dehydrogenase deficiency in Afghan populations: implications for treatment policy in the region. Malaria Journal 2013 12:230

\section{Submit your next manuscript to BioMed Central and take full advantage of:}

- Convenient online submission

- Thorough peer review

- No space constraints or color figure charges

- Immediate publication on acceptance

- Inclusion in PubMed, CAS, Scopus and Google Scholar

- Research which is freely available for redistribution

Submit your manuscript at www.biomedcentral.com/submit
C Biomed Central 